that since the end of the 1960s CERN has been either the leading or next to leading laboratory in the world of particle physics. But their claims to have developed a powerful new tool of assessment in scientific research are exaggerated and their use of it is misleading.

Originally, Martin and Irvine advertised their method as one of "converging partial indicators", based on the questionable assumption that if a variety of uncertain measures appear to agree, the result is reliable. Having applied this method to CERN and found that the indicators do not converge, they nonetheless continue by "interpreting" the non-convergence. Here they rely on an opinion poll of physicists, so the new method boils down to a peer review interpreted by social scientists.

The authors base their main conclusions on a selection of so-called "crucial" experiments. This is hardly the way a serious history of the extraordinary progress in particle physics over the past ten or fifteen years will eventually be written. Such an approach traps the unwary into supposing all other results are negligible, and is a travesty of the basis on which scientific understanding advances. CERN is given credit for the discovery of the weak neutral current, which opened the door for the Glashow-Salam-Weinberg unified theory of the electromagnetic and weak forces and set the stage for much of what has followed. But this discovery did not win a place in the "top eleven" selected by citation analysis.

The method clearly fails seriously in not giving proper weight to several other CERN results of fundamental and lasting importance. For example, experiments at the intersecting storage rings discovered that the strong interactions of the proton are due to point-like constituents; deep inelastic neutrino experiments showed that the weak interactions of the proton also involve point-like "partons" and, when put together with the electron scattering results from the electron linear accelerator (SLAC) at Stanford, demonstrated that the partons are quarks. High-energy neutrino experiments found departures from point-like scattering of a kind attributable to the effects of the quarkquark forces, providing early support for quantum chromodynamics, the now established theory of the strong interaction.

Nevertheless, it is an accepted view that over the decade 1969 to 1978 , US laboratories would have appeared more often in a list of top experiments than Europe. Why? Because Martin and Irvine have set themselves the objective of judging CERN, they have no device but to attribute this difference in performance to some inadequacy of managements and physicists at CERN. Unsupported by evidence, they even attribute what they regard as CERN's poorer performance to its being an international laboratory with scientific advisory commitees accountable to national interests. Anyone with the slightest acquaintance with the ways CERN's scientific programme is handled knows there is no such interference; in making this inference Martin and Irvine display such a total ignorance of how CERN actually works that it must invalidate any other conclusions they reach.

With all their great effort of hindsight, Martin and Irvine are yet blind to the single most significant factor in the EuropeUnited States comparison in the 1970s: the electron-positron collider (SPEAR) at Stanford, together with SLAC, was responsible for the major part of the difference in score on which they hang their case. All will agree that SPEAR was, supremely, the best type of machine for the physics of the 1970s; once this factor is allowed for, the differences are not significant.

No doubt, in retrospect, some decisions of the CERN management and actions of some physicists if differently made could have led to greater success. But this can be said of most scientific endeavours including those in the United States. What Irvine and Martin seem unable to understand, and what makes their analysis so misleading, is that in research of this nature, until the experiments are done, noone knows which is the best choice. This is why the "methodology" of Martin and Irvine could not have predicted the remarkable success of Stanford in the 1970s.

The authors conclude that after the 1970s, CERN's performance has improved. We are given no "explanation" for this, which the analysis of the preceding years would not have foretold. Their naivety makes any views they express irrelevant to the assessment of any future enterprise such as LEP.

University of Oxford,

J.H. MULVEY

Nuclear Physics Laboratory,

Keble Road,

Oxford OXI $3 R H, U K$

\section{Mental arithmetic}

SIR - I was surprised to read in David Singmaster's review ${ }^{1}$ of Steven B. Smith's book, The Great Mental Calculators, that the mental calculation of the square root of 51 is considered to be so difficult.

I have just calculated the answer to be 7.14142842854216; the last two digits should be 85 I believe. To perform this calculation I have to write down the number (in fact $510,000,000,000$ is easier, giving the required answer $\times 10^{5}$ ), and also write down and see my growing answer. I have been able to do this for quite some time, but have not developed the capacity, it being of somewhat limited use!

To calculate $1 / 97$ to 96 decimal places, another example given by Dr Singmaster, seems quite easy, and I can multiply threedigit numbers in my head (again, provided they are written down), with a fairly high success rate. From these examples, I could match Alexander Aitken, one of the prodigies cited in the review, but not George Bidder. Indeed I have never thought in terms of logs, as did Bidder Taylor or Maclaurin series presumably? However I would think that compound interest calculations could be achieved fairly easily by use of suitable binomial coefficients.

As a postdoctoral biochemist I rarely have any call to use such mathematical abilities. But I am astounded that they are thought to be so rare.

c/o Department of Medicine,

BRIAN COOPER

\section{University of Bristol,}

Bristol Royal Infirmary,

Bristol BS2 $8 H W, U K$

1. Nature 310, 79 (1984)

\section{Animal rights}

SIR - In his article "Animal rights lobby cashes in" (Nature 13 September p.95) Marcus Chown points out that it is more than a year since the Government White Paper on animal experiments appeared. Neither in your article nor in recent articles and letters in The Times is mention made of certain wording relating to pain which I believe may be responsible for misunderstanding and anxiety. Mr Chown quotes the present rule "if an animal is found to be suffering severe pain which is likely to endure, it shall at once be killed". That is not unfortunately the whole story. Every licence to experiment on living animals carries a list of conditions. The passage quoted is from paragraph 3(b) of these conditions. 3(a) states that if an animal is suffering pain which is either severe or is likely to endure, and if the main result of the experiment has been attained, the animal shall forthwith be painlessly killed (italics mine). It is this which gives rise to problems and leads those of a suspicious mind to say that there is always a let-out. An experimenter faced with the loss of valuable experimental material might be tempted to hang on for a few more days until the experiment has been completed, with consequent suffering. This wording must be clarified. An important purpose of future legislation must be to allay public suspicion or disquiet and there must be no suggestion of an easy escape. Paragraph 3(c) of the conditions states that if an Inspector believes that an animal is suffering "considerable" pain he can direct it to be painlessly killed. We thus have pain described as "severe" and "considerable". Since it is impossible to give a clear definition of pain in animals, these terms are of doubtful value. Nevertheless the more moderate animal welfare organizations are right on insisting on some clarification of the "pain condition" and some attempt to balance possible suffering against possible benefits is not unreasonable.

R. BARER

Department of A natomy and Cell Biology,

University of Sheffield,

Sheffield S1O 2TN, UK 